\title{
Detection of PIK3CA mutations in circulating free DNA in patients with breast cancer
}

\author{
Ruth E. Board • Andrew M. Wardley • J. Michael Dixon • Anne C. Armstrong • \\ Sacha Howell · Lorna Renshaw • Emma Donald • Alastair Greystoke • \\ Malcolm Ranson · Andrew Hughes $\cdot$ Caroline Dive
}

Received: 8 January 2010/Accepted: 12 January 2010/Published online: 28 January 2010

(C) Springer Science+Business Media, LLC. 2010

\begin{abstract}
Somatic mutations in PIK3CA (encoding a class I phosphoinositide 3 kinase (PI3K) subunit) modulate PI3K signalling to influence tumour behaviour and occur in up to $40 \%$ of breast cancers. Inhibitors of PI3K signalling are entering clinical trials, but the impact of PIKC $3 A$ mutation on tumour response has yet to be clarified. This study investigated the potential utility of circulating free DNA (cfDNA) as a source for PIK3CA mutation detection in patients with breast cancer. cfDNA extracted (QIAamp Virus spin kit) from blood and matched archival tumour from 46 patients with metastatic breast cancer and 30 patients with localised, operable breast cancer was assessed for hotspot PIK3CA mutations using Amplification Refractory Mutation System (ARMS ${ }^{\mathrm{TM}}$ ) allele-specific PCR and Scorpion probes. PIK3CA mutations were detected in
\end{abstract}

R. E. Board · A. Greystoke · M. Ranson · C. Dive ( $₫)$

Clinical and Experimental Pharmacology Group, Paterson

Institute for Cancer Research, University of Manchester,

Manchester M20 4BX, UK

e-mail: cdive@picr.man.ac.uk

R. E. Board - A. M. Wardley - A. C. Armstrong - S. Howell ·

A. Greystoke $\cdot$ M. Ranson

Cancer Research UK Department of Medical Oncology,

Christie Hospital NHS Foundation Trust, Wilmslow Road,

Manchester M20 4BX, UK

J. M. Dixon - L. Renshaw

Edinburgh Cancer Research Centre, Western General Hospital,

Crewe Road South, Edinburgh EH4 2XR, UK

J. M. Dixon · L. Renshaw

Breakthrough Research Unit, Edinburgh, UK

E. Donald · A. Hughes

AstraZeneca Pharmaceuticals, Alderley Park,

Cheshire SK10 4TG, UK
13/46 (28\%) plasma-derived and 10/46 (21\%) serumderived cfDNA samples from metastatic breast cancer patients. In 41 cases with matched tumour and plasmaderived cfDNA data, concordance (same mutation status in plasma and tumour) was $95 \%$. Where a PIK3CA mutation was present in tumour, the 'pick up' in plasma-derived cfDNA was $80 \%$. PIK3CA mutations were present in tumours from 14/30 (47\%) localised breast cancers, but no PIK3CA mutations were detected in matched cfDNA. These data demonstrate feasibility and potential utility of cfDNA for PIK3CA mutation detection in patients with metastatic breast cancer. Studies are underway to qualify PIK3CA mutation in cfDNA as a predictive biomarker allowing patient stratification in clinical trials of mechanism-based therapeutics that target PI3K signalling pathways.

Keywords PIK3CA mutations - Breast cancer . Circulating free DNA . Predictive biomarker

\section{Introduction}

Somatic mutations in the gene encoding the $\mathrm{p} 110 \alpha$ catalytic subunit of the class 1A PI3K (PIK3CA) were identified in human cancers in 2004 [1]. The majority of mutations occur at three hotspots: p.E542K and p.E545K at exon 9 and p.H1047R at exon 20. These mutations enhance the lipid kinase activity of PIK3CA as compared to wild-type and can lead to increased phosphorylation of AKT and resultant transformation of normal cells to tumour cells in in vitro and in vivo studies [2-4].

It is postulated that cancers driven by PIK3CA mutations will be more sensitive to therapeutic inhibition of the PI3K pathway, and this is a fertile area for ongoing drug development. Cell lines and tumour xenografts harbouring 
mutations in PIK3CA are more sensitive to inhibitors of the PI3K pathway $[5,6]$ and the dual PI3K/mTOR inhibitor NVPBEZ235 reverses lapatinib resistance due to hyperactivation of the PI3K pathway by PIK3CA mutations [7]. In this era of mechanism-based therapeutics, considerable emphasis is being placed on identification of those patients most likely to respond, a process requiring qualified predictive biomarkers. In keeping with this approach, clinical trials designs for novel PI3K inhibitors would optimally include a predictive biomarker allowing enrichment of patients whose tumours exhibit deregulated PI3K pathway signalling and thus, if this pathway is of functional importance, whom are most likely to respond. $P I K 3 C A$ is mutated in a range of tumour types including up to $40 \%$ of breast cancers [8-12]. In breast cancer, emerging evidence suggests that $P I K 3 C A$ mutations are predictive of response to HER2 targeted agents: the HER2 overexpressing cell line BT-474 is rendered insensitive to the HER2 antibody trastuzumab when transduced with an active mutant of $P I K 3 C A$ and a shorter progression free survival was observed in patients with $P I K 3 C A$-mutated breast cancers treated with trastuzumab compared to those with wild-type tumours [13]. The potential utility of hotspot PIK3CA mutations as a predictive biomarker for drug development is thus gaining impetus.

Mutation status in tumours is assessed by analysis of DNA extracted from archival tumour tissue samples. This procedure is not without technical and logistical obstacles, particularly in multi-site, multi-national trials. Tumour biopsy material is not always readily available, even in patients with a more accessible tumour such as breast cancer. Furthermore, the limited and degraded amounts of DNA extracted from tumour biopsies and formalin-fixed paraffin-embedded (FFPE) tissues present inherent technical challenges for mutation detection. If the response to a therapeutic agent is dependent on the presence or absence of a particular DNA mutation, then the availability and quality of tumour-derived DNA for mutation analysis becomes critically important.

An alternative source of tumour-derived DNA is cell free or circulating free DNA (cfDNA). Small amounts of cfDNA $(\sim 1 \mathrm{ng}$ DNA/ml) circulate in the plasma of healthy individuals and approximately 4-fold greater levels of cfDNA are detected in cancer patients [14]. cfDNA can be extracted from plasma and/or serum, a less invasive and more readily accessible source of tumour DNA for mutation detection. Previous studies demonstrated that the feasibility of detecting tumour specific mutations in cfDNA from patients with cancer, including detection of epidermal growth factor receptor mutations in patients with non small cell lung cancer $[15,16], K R A S$ mutations in patients with pancreatic and colorectal cancers [17] and $B R A F$ mutations in patients with melanoma [18, 19].

We previously reported a novel assay to detect $P I K 3 C A$ mutations [20]. The aim of the present study was to assess the feasibility of $P I K 3 C A$ mutation detection in cfDNA from plasma or serum from patients with metastatic or operable breast cancer and to compare the cfDNA results with the PIK3CA mutation status of available matched tumours. Specifically, the $\%$ concordance for PIK3CA mutation (i.e. the same mutation status in tumour DNA and cfDNA) and the \% 'pick up' (where a mutation exists in tumour and is found in plasma/serum) were sought. The comparison of cfDNA from plasma or from serum was also explored. The results of this exploratory study will inform judgement on the potential clinical utility of $P I K 3 C A$ mutation detection cfDNA in this disease setting alongside factors of clinical sample availability, logistics and cost.

\section{Materials and methods}

\section{Patient samples}

Blood and archival tumour samples were obtained from patients with metastatic breast cancer treated at the Christie Hospital, Manchester, UK. Plasma, tumour samples and germ-line DNA from patients with operable breast cancer were obtained from the Edinburgh Research Cancer Centre, Edinburgh, Scotland, UK. Tissue was collected and stored by Edinburgh Cancer Research Centre from a grant supplied by Cancer Research UK. Research was conducted according to Good Clinical Practice and the Declaration of Helsinki. All patients provided written informed consent prior to participation.

\section{Sample processing and DNA extraction}

cfDNA was extracted from $1 \mathrm{ml}$ plasma or serum using a QIAamp MinElute Virus Spin Kit (Qiagen, Valencia, CA) according to manufacturer's instructions with the following modifications: $3 \mu \mathrm{g}$ tRNA (Sigma, Stockholm, Sweden), $125 \mu \mathrm{l}$ kit proteinase and $1 \mathrm{ml}$ kit lysis buffer were added to each $1 \mathrm{ml}$ sample of serum/plasma. Following $1 \mathrm{~h}$ incubation at $55^{\circ} \mathrm{C}, 1,250 \mu \mathrm{l}$ of $100 \% \mathrm{v} / \mathrm{v}$ ethanol was added and each sample was filtered through a MinElute column in aliquots until the sample was exhausted. Following standard wash procedures (according to manufacturer's instruction), the DNA was eluted twice in $40 \mu$ elution buffer. For analysis of tumour samples, hemotoxylin and eosin-stained slides were reviewed by a pathologist to confirm the presence of breast cancer cells within the section. DNA was extracted from $40 \mu \mathrm{m}$ unstained sections of FFPE samples by digestion in proteinase $\mathrm{K}$ (Fluka, Buchs, Switzerland) for $48 \mathrm{~h}$, boiling in 5\% chelex (Sigma-Aldrich Company Ltd, Dorset, UK) and chloroform phase extraction with ethanol precipitation [21]. 
PIK3CA mutation analysis

The four most common mutations in the PIK3CA gene, namely p.H1047R, p.H1047L, p.E545K and p.E542K, were assessed using Amplification Refractory Mutation System (ARMS) primers and Scorpion probes as previously described [20]. All assays were performed in duplicate.

Biostatistical analysis

Analysis was performed using a StatsDirect package (version 1.9.7; StatsDirect statistical software. England: StatsDirect Ltd. 2008). Fisher's exact test was used to calculate associations between clinical parameters and mutation status. Two sided $P$ values $<0.05$ were considered statistically significant. $95 \%$ confidence intervals (CI) were calculated using STATA (version 9.2; StataCorp. 2005. Stata Statistical Software: Release 9. College Station, TX: StataCorp LP).

\section{Results}

Patient characteristics

Fifty patients with metastatic breast cancer were enrolled at the Christie Hospital between October 2007 and November 2008. Thirty patients with operable breast cancer were enrolled at the Edinburgh Cancer Centre between May 2008 and September 2008. The average age of patients with metastatic disease was 59 years (range 43-79 years). In total 46 of the 50 patients with advanced disease were eligible for evaluation of PIK3CA mutations in cfDNA and tumours. Two patients withdrew consent and a further two patients were found to have poor venous access which resulted in insufficient blood collection for analysis. The average age of patients with operable disease was 64 years (range 39-88 years). The histological characteristics of the tumours are shown in Table 1.

PIK3CA mutation analysis on tumour samples

\section{Operable breast cancer patients}

Of 30 tumour samples that were available from patients with operable breast cancer 14 (47\%) expressed PIK3CA mutations (8 p.H1047R, 1 p.H1047L, 4 p.E545K and 1 p.E542K). All $P I K 3 C A$ mutation positive cases were oestrogen receptor (ER) positive. No PIK3CA mutations were detected in the 6 ER negative cases. This was statistically significant by Fisher's exact test $P=0.0185$. PIK3CA mutations were seen more often in grade 1/2 tumours compared to grade 3 tumours (Fisher's exact test $P=0.0395$ ). There were no associations
Table 1 Characteristics of breast cancers from patients enrolled into study to detect PIK3CA mutations in cfDNA

\begin{tabular}{|c|c|c|}
\hline & $\begin{array}{l}\text { Operable breast } \\
\text { cancer }(n=30) \\
\mathrm{N}(\%)\end{array}$ & $\begin{array}{l}\text { Advanced breast cancer } \\
(n=49 \text { tumours from } \\
n=46 \text { patients) } \mathrm{N}(\%)\end{array}$ \\
\hline \multicolumn{3}{|l|}{ Histology } \\
\hline IDC & $26(87)$ & $40(82)$ \\
\hline ILC & $2(7)$ & $7(14)$ \\
\hline Mixed & 0 & $2(4)$ \\
\hline Other & $1(3)$ & 0 \\
\hline Unknown & $1(3)$ & 0 \\
\hline \multicolumn{3}{|l|}{ Grade } \\
\hline I & $5(17)$ & $3(6)$ \\
\hline II & $16(53)$ & $14(29)$ \\
\hline III & $9(30)$ & $27(55)$ \\
\hline Unknown & 0 & $5(10)$ \\
\hline \multicolumn{3}{|c|}{ Oestrogen receptor } \\
\hline Positive & $24(80)$ & $37(76)$ \\
\hline Negative & $6(20)$ & $12(24)$ \\
\hline \multicolumn{3}{|l|}{ HER2 Status } \\
\hline Positive $(>3+)$ & $3(10)$ & $12(24)$ \\
\hline Negative & $27(90)$ & $37(76)$ \\
\hline \multicolumn{3}{|l|}{ Size } \\
\hline$<2 \mathrm{~cm}$ & $16(53)$ & $6(12)$ \\
\hline $2-5 \mathrm{~cm}$ & $12(40)$ & $20(41)$ \\
\hline$>5 \mathrm{~cm}$ & $2(7)$ & $9(18)$ \\
\hline Not applicable & 0 & $11(22)$ \\
\hline Unknown & 0 & $3(6)$ \\
\hline \multicolumn{3}{|l|}{ Lymph node } \\
\hline Positive & $4(13)$ & $26(50)$ \\
\hline Negative & $25(83)$ & $10(20)$ \\
\hline Not applicable & 0 & $11(22)$ \\
\hline Unknown & $1(3)$ & $2(7)$ \\
\hline
\end{tabular}

Note: Percentages may not equal $100 \%$ due to rounding

Abbreviations: IDC invasive ductal carcinoma, ILC invasive lobular carcinoma

between HER2 status ( $n=3$ cases HER2 $3+$ ) or lymph node status ( $n=4$ cases lymph node positive) and PIK3CA mutations. No PIK3CA mutations were detected in cfDNA of these patients. Importantly, no germ-line PIK3CA mutations were detected in DNA from patients with operable disease, confirming the somatic nature of the mutation in the mutation positive tumour samples. The results of PIK3CA analyses in patients with operable breast cancer are reported in Table 2 (Fig. 1).

\section{Metastatic breast cancer}

Forty-five tumour samples were available from 43 patients with metastatic breast cancer. Of these, nine samples were biopsy only samples (1 liver, 1 skin, 1 axillary and 6 breast 
Table 2 Summary of PIK3CA mutation analysis in tumour, cfDNA and germ-line DNA of patients with advanced and operable breast cancer

\begin{tabular}{|c|c|c|c|c|c|c|}
\hline \multirow{2}{*}{$\begin{array}{l}P I K 3 C A \text { mutations } \\
\mathrm{N}(\%)\end{array}$} & \multicolumn{3}{|c|}{ Advanced breast cancer } & \multicolumn{3}{|c|}{ Operable breast cancer } \\
\hline & $\begin{array}{l}\text { Tumour } \\
(n=45)\end{array}$ & $\begin{array}{l}\text { Plasma cfDNA } \\
(n=46)\end{array}$ & $\begin{array}{l}\text { Serum cfDNA } \\
(n=46)\end{array}$ & $\begin{array}{l}\text { Tumour } \\
(n=30)\end{array}$ & $\begin{array}{l}\text { Plasma cfDNA } \\
(n=26)\end{array}$ & $\begin{array}{l}\text { Germ-line DNA } \\
(n=30)\end{array}$ \\
\hline p.H1047R & $2(4)$ & $4(9)$ & $4(9)$ & $8(27)$ & 0 & 0 \\
\hline p.H1047L & $1(2)$ & $2(4)$ & $2(4)$ & $1(3)$ & 0 & 0 \\
\hline p.E545 K & $5(11)$ & $6(13)$ & $3(7)$ & $4(13)$ & 0 & 0 \\
\hline p.E542 K & $2(4)$ & $1(2)$ & $1(2)$ & $1(3)$ & 0 & 0 \\
\hline Total & $10(23)$ & $12(28)$ & $10(21)$ & $14(47)$ & 0 & 0 \\
\hline
\end{tabular}

Fig. 1 Schema summarising PIK3CA mutation analysis on tumour and cfDNA in patients with advanced breast cancer

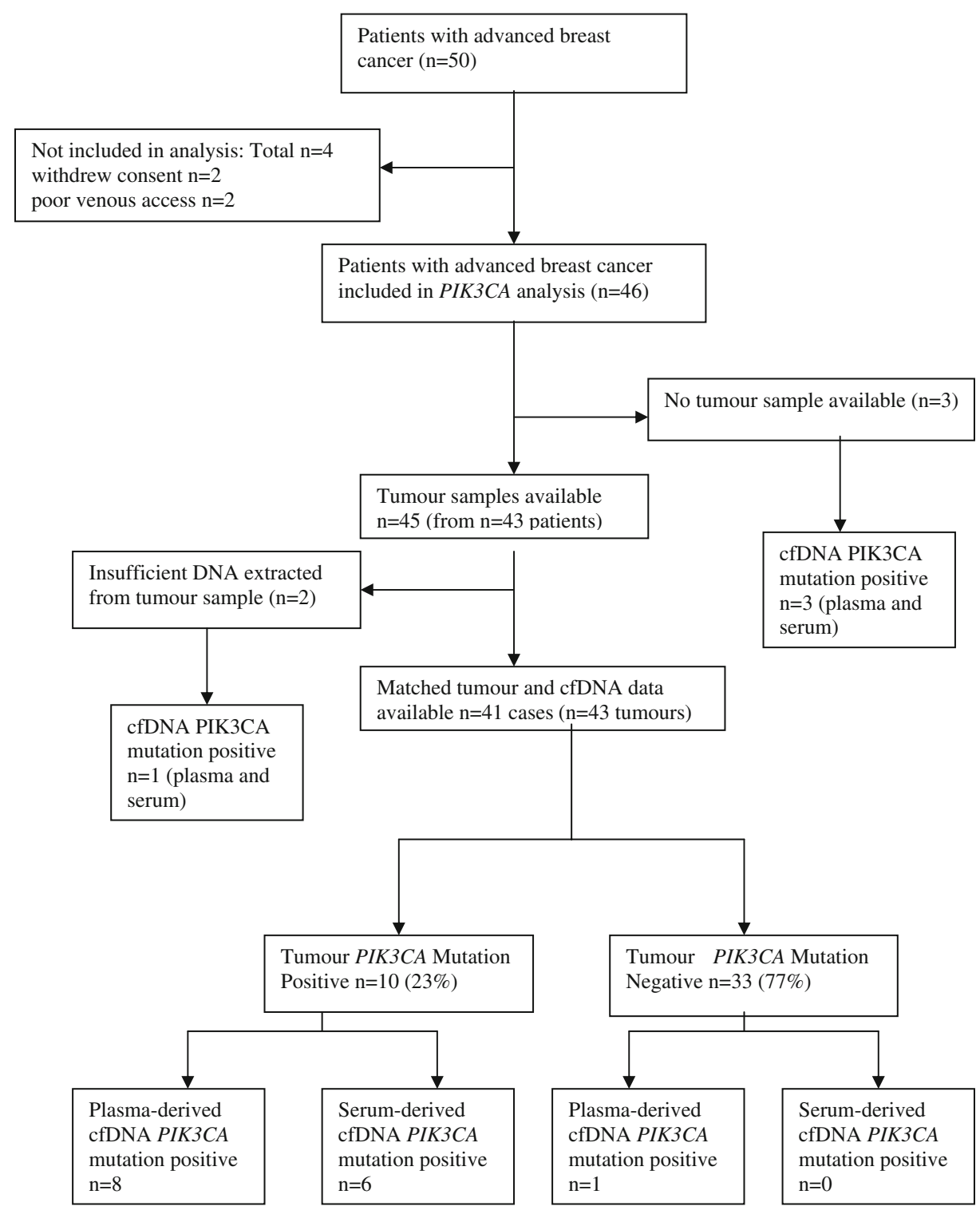

Plasma-derived cfDNA $P I K 3 C A$ analysis concordance rate with tumour $95 \%$, pick up rate $80 \%$ Serum-derived cfDNA PIK3CA analysis concordance rate with tumour $88 \%$, pick up rate $60 \%$ 
biopsies), the remainder were samples taken during primary breast surgery. In 11 cases, the tumour sample was taken at the same time as diagnosis of metastatic disease, either as a diagnostic biopsy $(n=9)$ or during primary surgery with metastatic disease diagnosed immediately post-operatively $(n=2)$. In three cases, tumour samples were unavailable; in two cases, the samples were lost; and in 1 case, initial surgery was performed outside the UK.

DNA was extracted from the 45 available tumour samples and sufficient DNA was extracted for analysis in 43/45 cases. In two cases, when biopsy material only was available, insufficient DNA was extracted for successful PIK3CA analysis. The loss of five cases from the original 48 tumours $(10 \%)$ illustrates the difficulty often experienced in obtaining sufficient number and quality of tumour biopsies for clinical research.

PIK3CA mutations were detected in 10 (23\%) of the 43 breast cancer samples (2 p.H1047R, 1 p.H1047L, 5 p.E545K and 2 p.E542K). There were no statistically significant associations between the presence of PIK3CA mutations and tumour grade, hormone receptor status, HER2 status or lymph node spread in this group of tumours. PIK3CA mutations were detected in plasmaderived cfDNA from 13 of $46(28 \%)$ patients with metastatic breast cancer (4 p.H1047R, 2 p.H1047L, 6 p.E545K and 1 p.E542K). Identical PIK3CA mutations were detected in serum-derived cfDNA from $10(21 \%)$ patients. In three cases, p.E545K PIK3CA mutations were detected in plasma-derived cfDNA, but not in the matched serum sample suggesting that plasma was the better matrix for this approach. The results of PIK3CA analysis in metastatic breast cancer patients are shown in Table 2 .

\section{Comparison of PIK3CA mutation detection}

results from tumour and cfDNA

Of the cases with metastatic breast cancer eligible for analysis $(n=46)$, matched tumour and plasma data was available for 41 cases. In four cases of missing tumour data, PIK3CA mutations were detected in cfDNA extracted from both plasma and serum. Of the 10 tumour samples with PIK3CA-detected mutations, PIK3CA mutations were detected in the matched plasma-derived cfDNA of eight cases and serum-derived cfDNA in six cases. In one case, a PIK3CA mutation was detected in cfDNA from plasma where the tumour DNA had no PIK3CA mutation detected. In this case, histological evaluation of the tumour sample revealed mainly ductal carcinoma in situ (DCIS) with no invasive component.

Thus, in 41 cases with matched tumour and cfDNA data, the concordance was $95 \%$ (95\% CI: 83-99\%) for plasmaderived cfDNA and $88 \%$ (95\% CI: $73-95 \%$ ) for serumderived cfDNA. If a PIK3CA mutation was present in the tumour DNA the 'pick up' in cfDNA was $80 \%$ (95\% CI: 44-97\%) for plasma-derived cfDNA and 60\% (95\% CI: 26-88\%) for serum-derived cfDNA.

\section{Discussion}

This study sought to determine the feasibility and potential clinical utility of PIK3CA mutations detection in cfDNA in breast cancer patients. The study is important given the relatively high percentage of human tumours, including breast cancer, that exhibit PIKC $3 A$ mutations and is timely due to the approach of novel PI3K pathway inhibitors in early clinical trials that will most likely require patient stratification for optimal clinical drug development. Our contention is that if PIKC $3 A$ mutations can be reliably assessed in cfDNA, blood sampling will be easier, cheaper and less invasive for cancer patients than tumour biopsy and further studies to qualify PIK3CA mutation detection in cfDNA as a predictive biomarker in breast cancer clinical trials of PI3K pathway targeted therapies should be implemented.

In this exploratory study, PIK3CA mutations were detected in plasma-derived cfDNA from 13/46 (28\%) patients with metastatic breast cancer. This is the first report to demonstrate detection of PIK3CA mutations in cfDNA. In three cases, mutations were not detected in matched serum-derived cfDNA. In two of these cases, a PIK3CA mutation was confirmed in tumour. We hypothesise that the reason for the higher mutation detection rate in plasma compared to serum relates to lysis of white cells during the clotting process. This increases levels of wild-type DNA sequences within a sample, hence, reducing the proportion of mutated sequences making detection of tumour associated cfDNA alterations less sensitive. In the third case, histological evaluation of the tumour specimen revealed mainly DCIS with little invasive cancer making mutation analysis less informative, and highlighting the issue of heterogeneity within tumour.

In contrast to the results from patients with metastatic disease, but perhaps not unexpectedly, no PIK3CA mutations were detected in plasma-derived cfDNA from patients with operable breast cancer. Less tumour DNA is present in the circulation in early stage disease compared to later stage cancers [22]. In colorectal cancer, the amount of tumour DNA in the circulation increases from $0.15 \%$ in Dukes stage B tumours to over $3 \%$ in more advanced disease [22]. Previous studies have demonstrated that a 'stage-dependency' effect for mutation detection cfDNA where the detection of cfDNA mutations is lower in patients with stage 1 or 2 disease compared to more advanced disease $[19,23]$. This is not considered to present a significant barrier to clinical utility of cfDNA for mutation analysis, as in the local setting, tumour material from 
surgery of curative intent should provide sufficient tumour material directly for definitive mutation analysis. It is also more likely that patients entering early clinical trials of PI3Kdirected therapies will have failed standard care options and have advanced disease, therefore more likely to have sufficient cfDNA from the tumour for mutation analysis.

A concordance for $95 \%$ for PIK3CA mutations in plasma-derived cfDNA compared to tumour DNA in patients with advanced breast cancer was extremely encouraging and now merits further study in larger patient cohorts. In four cases, PIK3CA mutations were detected in cfDNA when no tumour result was available (unavailable tumour samples or insufficient DNA extractable), highlighting the common problem associated with obtaining archival tumour samples and serving as an exemplar of how cfDNA could be utilised in the absence of tumour data to negate the need for further tumour biopsies.

PIK3CA mutations were detected in plasma-derived cfDNA in 8 of 10 cases with tumour PIK3CA mutations $(80 \%$; 95\% CI: 44-87\%). This 'pick up' in plasma is higher than that observed in most previous studies of mutation detection in cfDNA which report pick up rates of around 50\%. This favourable discrepancy between the current and previous reports raises some important questions: are mutant cfDNA fragments present in all patients, but currently applied technologies insufficiently sensitive to detect them, or do differences in tumour location and biology and/or host factors mean that cfDNA mutations are only shed (or shed at a higher level) in certain patients and/or tumour types? Perhaps certain tumours 'shed' more cfDNA than others, e.g. there could be greater cfDNA-mutated sequences from some tumours or tumour types due to tumour location, size, vascularity or apoptotic rate. It is possible that mutations are more easily detected in cfDNA of patients with breast cancer, certainly very high levels of circulating tumour cells (CTCs) are reported for patients with metastatic breast cancer compared to metastatic NSCLC or pancreatic cancer, perhaps reflecting different behaviours of different tumour types with respect to access of tumour cells or molecules shed from tumour cells into peripheral blood [24]. Further studies are required to answer these questions as translational research technologies continually improve.

In this series, there were two cases where PIK3CA mutations were detected in tumour samples, but not in cfDNA; although anecdotal, these cases are interesting. In both cases, the patient had two previous primary breast tumours and, given the absence of further tissue biopsies of metastatic lesions, it is difficult to predict which of the previous tumours were responsible for the metastatic spread and it though speculative, the absence of PIK3CA mutations in cfDNA may reflect the fact that a PIK3CA wild-type tumour was the cause of the metastatic disease. These two interesting cases highlight where a comparison between mutation status in tumour DNA and cfDNA might provide additional information on tumour behaviour.

There was only a single example in this study of a cfDNAdetected mutation with a tumour sample negative for PIK3CA mutations. The sample analysed from the tumour consisted mainly of DCIS with little invasive disease on the section. The lack of invasive cancer in the tissue analysed is the most likely explanation for the discrepancy, and it is likely that a PIK3CA mutation was present in the invasive cancer, but this was not detected due to the small amount of invasive cancer in the sample. Previous studies with other mutations have also reported cases where mutations have been detected in cfDNA, but not in the tumour specimen and again highlight additional gathering of potentially important data with cfDNA analysis [18, 25].

As the clinical importance of $P I K 3 C A$ is defined, there will be an increasing need to be able to assess the PIK3CA mutation status of a patient's tumour. This is the first report of the detection of PIK3CA mutations in cfDNA from patients with cancer. Further work with increased patient cohort sizes should qualify cfDNA as a surrogate for tumour regarding PIK3CA mutation detection in these patients. The results from this study provide confidence that PIK3CA mutations can be detected in cfDNA of patients with advanced breast cancer. This approach could be applied to clinical trials of novel agents where $P I K 3 C A$ mutations may have a predictive role, e.g. in the development of novel PI3K or mTOR inhibitors. If this is confirmed retrospectively, a future aim would be to preselect patients prospectively for treatment or trial entry based on cfDNA mutation results which could be provided in real time (within $48 \mathrm{~h}$ ) replacing the rate limiting step awaiting tumour biopsy results or subjecting patients to further biopsies when historical tumour samples are unavailable or insufficient for analysis. Conceivably, mutation analysis in cfDNA could also serve as a pharmacodynamic marker of tumour response and disease relapse, if therapy results in its disappearance and reappearance, respectively. The high $\%$ concordance and pick up of PIKC $3 A$ mutations in cfDNA in advance breast cancer patients shown here is very encouraging, studies in other cancer types are now underway.

Acknowledgements Ruth Board was funded via a Cancer Research UK and AstraZeneca Clinical Pharmacology Fellowship.

\section{References}

1. Samuels Y, Wang Z, Bardelli A, Silliman N, Ptak J, Szabo S, Yan H, Gazdar A, Powell SM, Riggins GJ, Willson JK, Markowitz S, Kinzler KW, Vogelstein B, Velculescu VE (2004) High frequency of mutations of the PIK3CA gene in human cancers. Science 304(5670):554

2. Kang S, Bader AG, Vogt PK (2005) Phosphatidylinositol 3-kinase mutations identified in human cancer are oncogenic. Proc Natl Acad Sci USA 102(3):802-807 
3. Gymnopoulos M, Elsliger MA, Vogt PK (2007) Rare cancerspecific mutations in PIK3CA show gain of function. Proc Natl Acad Sci USA 104(13):5569-5574

4. Samuels Y, Diaz LA Jr, Schmidt-Kittler O, Cummins JM, Delong L, Cheong I, Rago C, Huso DL, Lengauer C, Kinzler KW, Vogelstein B, Velculescu VE (2005) Mutant PIK3CA promotes cell growth and invasion of human cancer cells. Cancer Cell 7(6):561-573

5. Ihle NT, Williams R, Chow S, Chew W, Berggren MI, PaineMurrieta G, Minion DJ, Halter RJ, Wipf P, Abraham R, Kirkpatrick L, Powis G (2004) Molecular pharmacology and antitumor activity of PX-866, a novel inhibitor of phosphoinositide-3kinase signaling. Mol Cancer Ther 3(7):763-772

6. Serra V, Markman B, Scaltriti M, Eichhorn PJ, Valero V, Guzman M, Botero ML, Llonch E, Atzori F, Di Cosimo S, Maira M, Garcia-Echeverria C, Parra JL, Arribas J, Baselga J (2008) NVPBEZ235, a dual PI3K/mTOR inhibitor, prevents PI3K signaling and inhibits the growth of cancer cells with activating PI3K mutations. Cancer Res 68(19):8022-8030

7. Eichhorn PJ, Gili M, Scaltriti M, Serra V, Guzman M, Nijkamp W, Beijersbergen RL, Valero V, Seoane J, Bernards R, Baselga J (2008) Phosphatidylinositol 3-kinase hyperactivation results in lapatinib resistance that is reversed by the mTOR/phosphatidylinositol 3-kinase inhibitor NVP-BEZ235. Cancer Res 68(22): 9221-9230

8. Saal LH, Holm K, Maurer M, Memeo L, Su T, Wang X, Yu JS, Malmstrom PO, Mansukhani M, Enoksson J, Hibshoosh H, Borg A, Parsons R (2005) PIK3CA mutations correlate with hormone receptors, node metastasis, and ERBB2, and are mutually exclusive with PTEN loss in human breast carcinoma. Cancer Res 65(7):2554-2559

9. Campbell IG, Russell SE, Choong DY, Montgomery KG, Ciavarella ML, Hooi CS, Cristiano BE, Pearson RB, Phillips WA (2004) Mutation of the PIK3CA gene in ovarian and breast cancer. Cancer Res 64(21):7678-7681

10. Levine DA, Bogomolniy F, Yee CJ, Lash A, Barakat RR, Borgen PI, Boyd J (2005) Frequent mutation of the PIK3CA gene in ovarian and breast cancers. Clin Cancer Res 11(8):2875-2878

11. Bachman KE, Argani P, Samuels Y, Silliman N, Ptak J, Szabo S, Konishi H, Karakas B, Blair BG, Lin C, Peters BA, Velculescu VE, Park BH (2004) The PIK3CA gene is mutated with high frequency in human breast cancers. Cancer Biol Ther 3(8):772-775

12. Lee JW, Soung YH, Kim SY, Lee HW, Park WS, Nam SW, Kim SH, Lee JY, Yoo NJ, Lee SH (2005) PIK3CA gene is frequently mutated in breast carcinomas and hepatocellular carcinomas. Oncogene 24(8):1477-1480

13. Berns K, Horlings HM, Hennessy BT, Madiredjo M, Hijmans EM, Beelen K, Linn SC, Gonzalez-Angulo AM, Stemke-Hale K, Hauptmann M, Beijersbergen RL, Mills GB, van de Vijver MJ, Bernards R (2007) A functional genetic approach identifies the PI3 K pathway as a major determinant of trastuzumab resistance in breast cancer. Cancer Cell 12(4):395-402
14. Leon SA, Shapiro B, Sklaroff DM, Yaros MJ (1977) Free DNA in the serum of cancer patients and the effect of therapy. Cancer Res 37(3):646-650

15. Kimura H, Kasahara K, Kawaishi M, Kunitoh H, Tamura T, Holloway B, Nishio K (2006) Detection of epidermal growth factor receptor mutations in serum as a predictor of the response to gefitinib in patients with non-small-cell lung cancer. Clin Cancer Res 12(13):3915-3921

16. Kimura H, Suminoe M, Kasahara K, Sone T, Araya T, Tamori S, Koizumi F, Nishio K, Miyamoto K, Fujimura M, Nakao S (2007) Evaluation of epidermal growth factor receptor mutation status in serum DNA as a predictor of response to gefitinib (IRESSA). Br J Cancer 97(6):778-784

17. Sorenson GD (2000) Detection of mutated KRAS2 sequences as tumor markers in plasma/serum of patients with gastrointestinal cancer. Clin Cancer Res 6(6):2129-2137

18. Yancovitz M, Yoon J, Mikhail M, Gai W, Shapiro RL, Berman RS, Pavlick AC, Chapman PB, Osman I, Polsky D (2007) Detection of mutant BRAF alleles in the plasma of patients with metastatic melanoma. J Mol Diagn 9(2):178-183

19. Daniotti M, Vallacchi V, Rivoltini L, Patuzzo R, Santinami M, Arienti F, Cutolo G, Pierotti MA, Parmiani G, Rodolfo M (2007) Detection of mutated BRAFV600E variant in circulating DNA of stage III-IV melanoma patients. Int J Cancer 120(11):2439-2444

20. Board RE, Thelwell NJ, Ravetto PF, Little S, Ranson M, Dive C, Hughes A, Whitcombe D (2008) Multiplexed assays for detection of mutations in PIK3CA. Clin Chem 54(4):757-760

21. Coombs NJ, Gough AC, Primrose JN (1999) Optimisation of DNA and RNA extraction from archival formalin-fixed tissue. Nucleic Acids Res 27(16):e12

22. Diehl F, Li M, Dressman D, He Y, Shen D, Szabo S, Diaz LA Jr, Goodman SN, David KA, Juhl H, Kinzler KW, Vogelstein B (2005) Detection and quantification of mutations in the plasma of patients with colorectal tumors. Proc Natl Acad Sci USA 102(45):16368-16373

23. Wang JY, Hsieh JS, Chang MY, Huang TJ, Chen FM, Cheng TL, Alexandersen K, Huang YS, Tzou WS, Lin SR (2004) Molecular detection of APC, K-ras, and p53 mutations in the serum of colorectal cancer patients as circulating biomarkers. World J Surg 28(7):721-726

24. Hayes DF, Cristofanilli M, Budd GT, Ellis MJ, Stopeck A, Miller MC, Matera J, Allard WJ, Doyle GV, Terstappen LW (2006) Circulating tumor cells at each follow-up time point during therapy of metastatic breast cancer patients predict progressionfree and overall survival. Clin Cancer Res 12(14 Pt 1):4218-4224

25. Ramirez JL, Sarries C, de Castro PL, Roig B, Queralt C, Escuin D, de Aguirre I, Sanchez JM, Manzano JL, Margeli M, Sanchez JJ, Astudillo J, Taron M, Rosell R (2003) Methylation patterns and K-ras mutations in tumor and paired serum of resected nonsmall-cell lung cancer patients. Cancer Lett 193(2):207-216 